\title{
Prevalence of Primary Open Angle Glaucoma among Patients with Diagnosis of Systemic Hypertension and Diabetes Mellitus: The Colombian Glaucoma Study
}

\author{
Carlos E. Rivera ${ }^{1 *}$, Erika Cantor ${ }^{2}$, Andres Castillo ${ }^{3}$, Alexander Martinez ${ }^{4}$, Lyle Newball ${ }^{5}$, \\ Juan C. Rueda ${ }^{6}$, Alejandro Valencia ${ }^{7}$, Sandra Belalcazar ${ }^{8}$, Tulio Cabal ${ }^{9}$, \\ Oscar Albis-Donado ${ }^{10}$, Fabian Mendez ${ }^{11}$ \\ ${ }^{1}$ Fundación Oftalmológica del Valle, Cali, Colombia \\ ${ }^{2}$ Institute of Statistics, Universidad de Valparaiso, Valparaiso, Chile \\ ${ }^{3}$ Department of Biology, Faculty of Natural \& Exact Sciences, Universidad del Valle, Cali, Colombia \\ ${ }^{4}$ Ophthalmology Residency Program, Universidad del Valle, Centro Médico Imbanaco, Cali, Colombia \\ ${ }^{5}$ Clínica Lynd Newball, San Andrés Islas, Colombia \\ ${ }^{6}$ FOSCAL Internacional, Bucaramanga, Colombia \\ ${ }^{7}$ Glaucoma Unit Medellin Colombia \\ ${ }^{8}$ Fundación Oftalmológica Nacional, Bogotá, Colombia \\ ${ }^{9}$ Clínica Visión Cabal, Buga, Colombia \\ ${ }^{10}$ Visual Sense, Private Practice, Mexico City, Mexico \\ ${ }^{11}$ School of Public Health, GESP Group, Universidad del Valle, Cali, Colombia \\ Email: *carlosriverahoyos@gmail.com
}

How to cite this paper: Rivera, C.E., Cantor, E., Castillo, A., Martinez, A., Newball, L., Rueda, J.C., Valencia, A., Belalcazar, S., Cabal, T., Albis-Donado, O. and Mendez, F. (2020) Prevalence of Primary Open Angle Glaucoma among Patients with Diagnosis of Systemic Hypertension and Diabetes Mellitus: The Colombian Glaucoma Study. Open Journal of Ophthalmology, 10, $99-114$.

https://doi.org/10.4236/ojoph.2020.102012

Received: March 12, 2020

Accepted: April 26, 2020

Published: April 29, 2020

\begin{abstract}
Objective: To establish the prevalence and risk factors of Primary Open Angle Glaucoma (POAG) among patients with Systemic Hypertension (SH) and Diabetes Mellitus (DM) in six cities of Colombia. Methods: A cross-sectional study among hypertensive and diabetic patients was conducted in Colombia. This study included 2067 subjects older than 50 years of age diagnosed with SH and/or DM. Participants underwent a complete ophthalmic examination including intraocular pressure (IOP) measurement by Goldmann tonometry and blood pressure measurement. The glaucoma diagnosis was confirmed by structural and functional evidence. Interviews and standardized questionnaires were used to evaluate participants' lifestyle and other health conditions. Results: Among participants with DM/SH, 142 cases of POAG were confirmed for a prevalence of 5.6\% [95\% CI: 4.6 - 6.6], while 9.1\% were glaucoma suspects [ $95 \%$ CI: $7.8 \%-10.4 \%$ ]. The majority of confirmed cases $(77.5 \%)$ were undiagnosed. The prevalence of POAG was significantly higher with male gender, greater age, and diastolic blood pressure $>90 \mathrm{mmHg}$ as risk factors.
\end{abstract}


Copyright (C) 2020 by author(s) and Scientific Research Publishing Inc. This work is licensed under the Creative Commons Attribution International License (CC BY 4.0).

http://creativecommons.org/licenses/by/4.0/
Conclusion: We found a high prevalence of POAG in patients with adequate $\mathrm{SH}$ and DM care in a novel Latino population. We also found great unawareness of the disease in this population. Our results have potentially enormous public health implications for Colombia and other Latino populations.

\section{Keywords}

Open Angle-Glaucoma, Glaucoma, Systemic Hypertension, Diabetes Mellitus

\section{Introduction}

World-wide, the prevalence of glaucomatous optic neuropathy is estimated to be around 60 million cases, which implies there could be close to 8.4 million cases of blindness from glaucoma. The burden of glaucoma is expected to increase significantly, to an estimated 80 million people with glaucomatous optic neuropathy and 11.2 million blind by 2020 [1].

Many epidemiologic studies have been conducted in African-derived populations and non-Hispanic whites in the US and worldwide on the prevalence of glaucoma [2]-[11]. However, there have been relatively few studies in the Latino population [10] [12], especially in Colombia, South America, so the prevalence of glaucoma and its association with systemic hypertension $(\mathrm{SH})$ and diabetes mellitus (DM) is currently unknown in this setting.

Diabetes Mellitus (DM) has been previously associated with glaucoma prevalence, specifically with Primary Open Angle Glaucoma (POAG), but results vary widely and some are even contradictory: some find a direct relationship between DM and POAG [12] [13] [14] [15] [16]; others report a relationship between glucose levels and intraocular pressure (IOP) but do not show a relationship between DM and POAG [17]; and finally, there are some studies that find no relationship [18] [19]. The proposed causal relationship is that the known microvascular changes from DM may facilitate or induce glaucoma damage independently or by the coexistence with elevated IOP and other comorbidities.

Blood pressure (BP) and IOP have a direct proportional relationship, where, the higher the BP, the greater the IOP [20]. In contrast, an association between patients with treated $\mathrm{SH}$ who present nocturnal hypotension and glaucoma progression has also been reported. Still, there is no convincing explanation for the exact mechanism by which SH and glaucoma are related [21].

The aim of the study is to establish the prevalence of glaucoma in patients over 50 years of age and diagnosis of SH and/or DM in 6 cities of Colombia, and report on related risk factors.

\section{Materials and Methods}

\subsection{Study Design}

This is a cross-sectional study of hypertensive and diabetic patients of six cities in Colombia (Bogotá, Buga, Bucaramanga, Cali, Medellin, and San Andres), 
conducted from September 2014 to January 2019. At enrollment, individuals were $\geq 50$ years of age and were treated with antihypertensive and/or anti-diabetic medications for at least 1 year. The diagnosis of DM and SH were verified according to the guidelines for each disease [22] [23]. Patients with severe associated comorbidities (renal failure, congestive heart failure, sleep apnea, autoimmune diseases with biological therapy), previous intraocular surgery (trauma, retinal detachment, complicated cataract surgery, macular degeneration or maculopathy) or congenital ocular pathology (e.g., coloboma) were excluded. All participants were selected from SH and DM control programs. The Universidad del Valle Review Board approved this study (Approval Code 030-014), all participants signed an informed consent form. This research was conducted according to the tenants of the Declaration of Helsinki.

\subsection{Procedures}

Interviews and questionnaires were used to evaluate factors related to participants' lifestyle, and other health conditions, including socioeconomic status, associated comorbidities, education and nutrition. Family history of glaucoma and knowledge of the disease were also recorded. Physical activity was measured using the International Physical Activity Questionnaire (IPAQ). In addition, a physical examination was performed that included measurement of height, weight, abdominal circumference, heart rate and systolic (SBP) and diastolic blood pressure (DBP).

Blood pressure (BP) was measured in sitting position after 5 minutes of rest, using a sphygmomanometer (Welch Allyn, New York, U.S.). The cut-off values of BP were defined according to the guidelines for the management of Arterial hypertension of the European Society of Hypertension (ESH) [23]. High BP was defined as $\mathrm{SBP}>140 \mathrm{~mm} \mathrm{Hg}$ or DBP $>90 \mathrm{~mm} \mathrm{Hg}$. Low BP was defined as $\mathrm{SBP}<$ $110 \mathrm{~mm} \mathrm{Hg}$ and DBP $<60 \mathrm{~mm} \mathrm{Hg}$. Mean arterial BP (MABP) was calculated as $(1 / 3) \mathrm{SBP}+(2 / 3) \mathrm{DBP}$. The Ocular Perfusion Pressure (OPP) was defined as $2 / 3$ MABP-IOP. The highest IOP value between the two eyes was used to calculate OPP.

\subsection{Ophthalmic Evaluation}

Each participant underwent a complete ophthalmologic examination, including visual acuity, refraction, slit-lamp examination, intraocular pressure and pachymetry measurements. The IOP measurement was obtained from the average of three values by Goldmann tonometry. Gonioscopy was performed in a dark room using a 4-mirror goniolens (Ocular Instruments Inc., Bellevue, WA) in primary position, with a slit beam less than $2 \mathrm{~mm}$ in height, followed by a dilated funduscopic examination with a 78 diopter (D) lens for evaluating the optic disc, (Ocular Instruments Inc., Bellevue, WA). Central corneal thickness (CCT) was calculated based on the average of three consecutive measurements using a PachPen handheld pachymeter (Accutome, Inc., Pennsylvania, USA). 
In suspected cases of glaucoma, the diagnosis was confirmed using visual field (VF) test with the 24-2 Swedish Interactive Threshold Algorithm (Humphrey, Carl Zeiss Meditec, Inc) and optic nerve photos with a DRS camera (digital retinography system, Centervue, Fremont, CA, USA). Glaucomatous eyes had to have at least 2 consecutive, reliable, and repeatable standard automated perimetry examinations with either a pattern standard deviation (PSD) outside the 95\% normal limits or a glaucoma hemifield test result outside normal limits. Reliable visual fields had rates of false-positives, fixation losses and false-negative errors of $20 \%$ or less to be included. Trained glaucoma specialists performed the examinations using standardized protocols.

\subsection{Diagnosis of Glaucoma}

Suspected and confirmed cases of glaucoma were defined according to the criteria specified by Foster et al. [24] confirmed glaucoma was defined as structural and functional evidence of glaucomatous damage in at least one eye that met the following criteria: 1) horizontal or vertical cup-disc ratio 0.7 (97.5th percentile), focal glaucomatous disc change (disc hemorrhage, notch of the neuroretinal rim, marked sloping of rim tissue, narrowest remaining rim of 0.1 disc diameter or less), cup/disc asymmetry 0.2 (97.5th percentile), associated with a glaucomatous VF defect; 2) horizontal or vertical cup-disc ratio 0.8 (99.5th percentile), focal glaucomatous disc change, asymmetry 0.3 (99.5th percentile) with absence of functional evidence of glaucomatous damage (if the subject could not satisfactorily complete the VF examination). Cases that did not meet all criteria were classified as suspected glaucoma. In addition, VF defects that were not explained by any other disease, like asymmetry across the horizontal midline, visual defects located in the mid-periphery or clustered in neighboring test points, were defined as compatible with the disease.

\subsection{Sample Size and Statistical Analysis}

Sample size calculation was based on expected prevalence of POAG of $8 \%$, a $95 \%$ confidence interval level and a precision of $2 \%$; based on these parameters the estimated sample size was of 1632 patients (hypertensive and/or diabetic), who were all recruited from SH and DM control programs.

The glaucoma prevalence was calculated as the ratio between the number of individuals with suspected or confirmed glaucoma and the number of individuals included in the study. Continuous variables were summarized with mean \pm standard deviation (SD) or median and interquartile range (IQR), while categorical variables were described with proportions.

The patients were divided into three groups according to the status of diagnosis of POAG: confirmed cases, suspected cases and those without glaucoma. Participants with angle-closed or secondary glaucoma were excluded for this analysis. The comparison between categories of continuous variables was performed using ANOVA or the Kruskal-Wallis tests. Binary and categorical characteristics were compared using a chi-square or Fisher's exact tests. A multinomial logistic 
regression model was applied to determine factors associated with POAG. Model selection was performed using a backward selection methodology; variables with $\mathrm{p}$ values $<0.20$ in bivariate analysis were included. Odds Ratios (OR) were estimated with $95 \%$ confidence interval and goodness-of-fit was evaluated using a likelihood ratio test and the smallest model deviance. A level of significance of 0.05 was used. All analyses were carried out using Stata $13^{\circledR}$ (STATA Corp, College Station, TX, USA).

\section{Results}

A total of 2085 subjects completed the interview and ophthalmologic examination, of which 18 were excluded because they met one or more exclusion criteria. The average age of the 2067 participants was $65.6 \pm 8.8$ years, $64.1 \%$ (1324) were female, $11.0 \%$ (228) had only DM, 59.6\% (1231) had only SH and 29.4\% (608) had both diseases. Of $2067 \mathrm{SH}$ and/or DM patients, 142 were identified with confirmed glaucoma and 226 subjects with suspected glaucoma (Figure 1). From 1902 participants who completed the question about the family history of glaucoma, 20.9\% (398) answered affirmatively. Of 142 participants who were identified as confirmed glaucoma in the present study, 22.5\% (32) had been previously diagnosed.

\subsection{Prevalence of Glaucoma}

Prevalence of confirmed POAG was 5.6\% [95\% CI: 4.6 - 6.0], with a higher prevalence observed among those with $\mathrm{SH}$ only. In addition, the proportion of suspected cases was higher among participants with DM only (Table 1). The prevalence of POAG according to age and sex is described in Table 2. Confirmed POAG was more frequent in men at all ages. A higher prevalence of suspected POAG was found in cases under 59 years and older than 80 years. The prevalence of confirmed POAG was higher in diabetic or hypertensive patients with time since diagnosis of more than 5 years, while suspected POAG was more frequent in patients with an evolution of the disease less than 5 years (Figure 2).

\subsection{Characteristics of Patients with or without POAG}

The sociodemographic and clinical characteristics of 2085 cases with and without POAG are described in Table 3. Confirmed cases of POAG were more frequent among men and above 70 years of age. The proportion of self-identified individuals as African-descendant was more frequent in cases with suspected POAG. As compared with cases without glaucoma, suspected and confirmed POAG cases reported high physical activity more frequently. No statistically significant differences were found between groups regarding marital status, level of education, alcohol consumption, smoking status, weight, height, body mass index (BMI) and abdominal circumference. The percentage of cases with a family history of glaucoma was significantly higher $(29.2 \%)$ in confirmed cases of POAG (Table 3 and Table 4). 
Significant differences were found in DBP, with a higher proportion of confirmed POAG cases with pressure $<60 \mathrm{~mm} \mathrm{Hg}$ or $>90 \mathrm{~mm} \mathrm{Hg}$. Self-reported autoimmune disease was more frequent in confirmed POAG. A higher proportion of cases with thyroid disease was found among patients without glaucoma, in comparison with suspected and confirmed POAG $(\mathrm{p}<0.05)$ (Table 3 and Table 4).

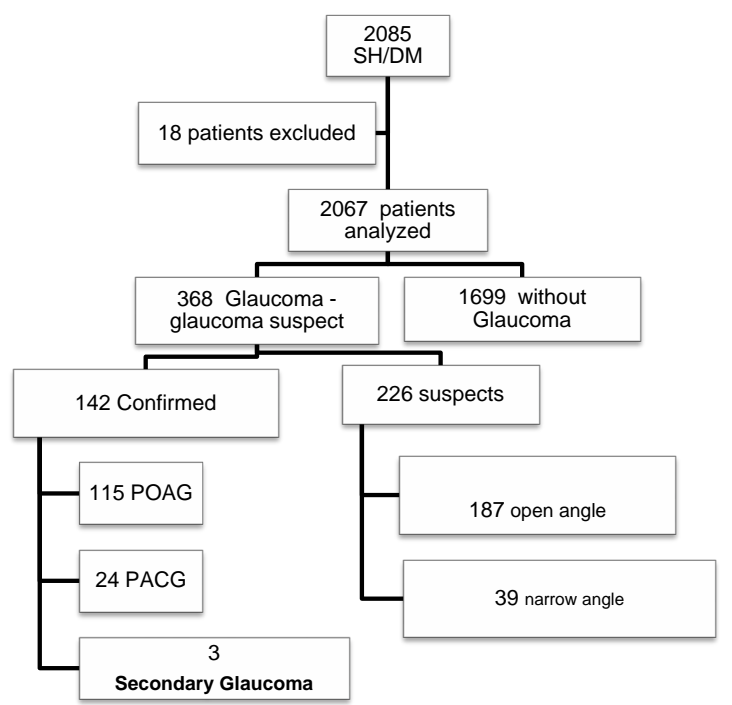

POAG: Primary open angle glaucoma; PACG Primary Angle Closed Glaucoma; SH: Systemic Hypertension; DM: diabetes Mellitus.

Figure 1. Flowchart of the study participants.

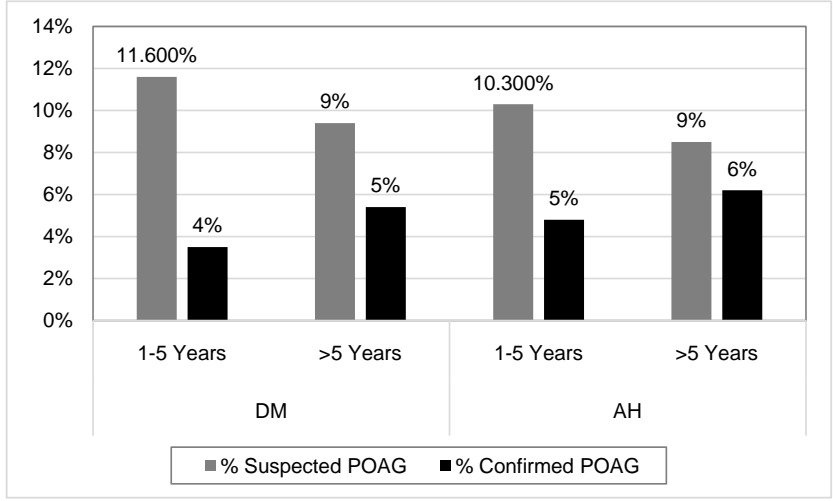

Figure 2. Prevalence of POAG according to time since diagnosis of $\mathrm{DM}$ or $\mathrm{SH}$.

Table 1. Prevalence of POAG.

\begin{tabular}{ccccc}
\hline Glaucoma & $\begin{array}{c}\text { Total } \\
\%[95 \% \mathrm{CI}]\end{array}$ & $\begin{array}{c}\mathrm{DM} \\
\%[95 \% \mathrm{CI}]\end{array}$ & $\begin{array}{c}\mathrm{SH} \\
\%[95 \% \mathrm{CI}]\end{array}$ & $\begin{array}{c}\mathrm{DM} / \mathrm{AP} \\
\%[95 \% \mathrm{CI}]\end{array}$ \\
\hline Confirmed & $5.6[4.6-6.6]$ & $4.8[2.4-8.5]$ & $6.2[4.9-7.7]$ & $4.4[2.9-6.4]$ \\
Suspect & $9.1[7.8-10.4]$ & $11.4[7.6-16.3]$ & $8.6[7.1-10.3]$ & $10.5[8.2-13.2]$ \\
Total & $14.6[13.1-16.2]$ & $16.3[11.7-21.5]$ & $14.9[12.9-16.9]$ & $13.5[10.9-16.5]$ \\
\hline
\end{tabular}

POAG: Primary open angle glaucoma; SH: Systemic Hypertension; DM: diabetes; CI: Confidence interval. 
Table 2. Prevalence of POAG according to sex and age.

\begin{tabular}{ccccccc}
\hline \multirow{2}{*}{ Age } & \multicolumn{3}{c}{ Confirmed POAG } & \multicolumn{3}{c}{ Suspected POAG } \\
\cline { 2 - 7 } & $\begin{array}{c}\text { Women } \\
\%[95 \% \mathrm{CI}]\end{array}$ & $\begin{array}{c}\text { Men } \\
\text { \% }[95 \% \mathrm{CI}]\end{array}$ & $\begin{array}{c}\text { Total } \\
\%[95 \% \mathrm{CI}]\end{array}$ & $\begin{array}{c}\text { Women } \\
\%[95 \% \mathrm{CI}]\end{array}$ & $\begin{array}{c}\text { Men } \\
\%[95 \% \mathrm{CI}]\end{array}$ & $\begin{array}{c}\text { Total } \\
\%[95 \% \mathrm{CI}]\end{array}$ \\
\hline $50-59$ & $2.7[1.5-4.9]$ & $6.8[3.9-11.6]$ & $4.0[2.7-5.9]$ & $9.5[7.0-12.8]$ & $11.9[7.9-17.6]$ & $10.2[8.0-13.0]$ \\
$60-69$ & $3.5[2.2-5.6]$ & $6.0[3.8-9.4]$ & $4.5[3.2-6.1]$ & $9.3[7.0-12.1]$ & $8.4[5.7-12.1]$ & $8.9[7.1-11.1]$ \\
$70-79$ & $6.8[4.5-10.1]$ & $11.3[7.7-16.1]$ & $8.6[6.5-11.3]$ & $7.4[5.0-10.8]$ & $8.1[5.2-12.5]$ & $7.7[5.7-10.2]$ \\
$>80$ & $5.6[2.3-12.8]$ & $8.9[3.3-21.6]$ & $6.7[3.5-12.4]$ & $10.1[5.3-18.4]$ & $11.1[4.6-24.2]$ & $10.4[6.3-16.9]$ \\
\hline
\end{tabular}

POAG: Primary open angle glaucoma; CI: Confidence interval.

Table 3. Sociodemographic, clinical and ocular characteristics of cases with and without POAG.

\begin{tabular}{|c|c|c|c|c|c|}
\hline Variable & $\begin{array}{c}\text { Confirmed } \\
(\mathrm{n}=115)\end{array}$ & $\begin{array}{l}\text { Suspect } \\
(\mathrm{n}=187)\end{array}$ & $\begin{array}{l}\text { No Glaucoma } \\
\qquad(\mathrm{n}=1699)\end{array}$ & $\mathrm{p}$ value & $\begin{array}{c}\text { Total } \\
(\mathrm{n}=2001)\end{array}$ \\
\hline \multicolumn{6}{|l|}{ Age n (\%) } \\
\hline $50-59$ & $23(20.0)$ & $59(31.5)$ & $480(28.3)$ & \multirow{4}{*}{0.018} & $562(28.1)$ \\
\hline $60-69$ & $36(31.3)$ & $72(38.5)$ & $664(39.1)$ & & $772(38.5)$ \\
\hline $70-79$ & $47(40.9)$ & $42(22.5)$ & $445(26.2)$ & & $42(22.5)$ \\
\hline$>80$ & $9(7.8)$ & $14(7.5)$ & $108(6.4)$ & & $14(7.5)$ \\
\hline \multicolumn{6}{|l|}{$\operatorname{Sex} \mathrm{n}(\%)$} \\
\hline Female & $56(48.7)$ & $118(63.1)$ & $1105(65.0)$ & \multirow{2}{*}{0.002} & $1279(63.9)$ \\
\hline Male & $59(51.3)$ & $69(36.9)$ & $594(34.9)$ & & $722(36.1)$ \\
\hline \multicolumn{6}{|l|}{ Race n (\%) } \\
\hline Non White-White (mestizo) & $95(82.6)$ & $133(71.1)$ & $1345(79.2)$ & \multirow{4}{*}{0.018} & $1573(78.6)$ \\
\hline Black & $5(4.3)$ & $16(8.6)$ & $81(4.8)$ & & $102(5.1)$ \\
\hline White & $10(8.7)$ & $28(14.9)$ & $233(13.7)$ & & $271(13.5)$ \\
\hline Other & $5(4.3)$ & $10(5.3)$ & $40(2.3)$ & & $55(2.7)$ \\
\hline \multicolumn{6}{|l|}{ Marital Status n (\%) } \\
\hline Other & $45(39.1)$ & $68(36.6)$ & $734(43.2)$ & \multirow{2}{*}{0.167} & $847(42.4)$ \\
\hline Married/Free Union & $70(60.9)$ & $118(63.4)$ & $964(56.8)$ & & $1152(57.6)$ \\
\hline \multicolumn{6}{|l|}{ Educational Level n (\%) } \\
\hline High school or less & $90(78.9)$ & $142(76.8)$ & $1257(75.2)$ & \multirow{2}{*}{0.621} & $1489(75.6)$ \\
\hline Other & $24(21.0)$ & $43(23.2)$ & $414(24.8)$ & & $481(24.4)$ \\
\hline \multicolumn{6}{|l|}{ Physical Activity n (\%) } \\
\hline Low or inactive & $69(60.0)$ & $108(57.7)$ & $1123(66.1)$ & \multirow{3}{*}{0.006} & $1300(64.9)$ \\
\hline Moderate & $33(28.7)$ & $57(30.5)$ & $474(27.9)$ & & $564(28.2)$ \\
\hline high & $13(11.3)$ & $22(11.8)$ & $102(6.0)$ & & $137(6.8)$ \\
\hline \multicolumn{6}{|l|}{ Smoker n (\%) } \\
\hline Non smoker & $63(55.3)$ & $114(61.0)$ & $1029(60.7)$ & \multirow{3}{*}{0.819} & \\
\hline Ex-smoker & $46(40.3)$ & $66(35.3)$ & $593(35.0)$ & & 1206 \\
\hline Smoker & $5(4.4)$ & $7(3.7)$ & $72(4.2)$ & & \\
\hline \multicolumn{6}{|l|}{ Alcohol n (\%) } \\
\hline Never & $81(71.1)$ & $127(69.0)$ & $1251(74.2)$ & \multirow{2}{*}{0.270} & $1459(73.5)$ \\
\hline With some frecuency & $33(28.9)$ & $57(31.0)$ & $436(25.8)$ & & $526(26.5)$ \\
\hline \multicolumn{6}{|l|}{ Processed meat n (\%) } \\
\hline High Consumption & $15(13.3)$ & $36(19.3)$ & $177(10.5)$ & \multirow{2}{*}{0.001} & $228(11.5)$ \\
\hline Low Consumption & $98(86.7)$ & $150(80.6)$ & $1508(89.5)$ & & $1756(88.5)$ \\
\hline \multicolumn{6}{|l|}{ Red meat n (\%) } \\
\hline High Consumption & $65(56.5)$ & $112(59.9)$ & $1024(60.3)$ & \multirow{2}{*}{0.720} & $1201(60.1)$ \\
\hline Low Consumption & $50(43.5)$ & $75(40.1)$ & $673(39.7)$ & & $798(39.9)$ \\
\hline \multicolumn{6}{|l|}{ Salt $\mathbf{n}(\%)$} \\
\hline Low Consumption & $106(93.8)$ & $179(95.7)$ & $1634(96.9)$ & \multirow{2}{*}{0.142} & $1919(96.6)$ \\
\hline High Consumption & $7(6.2)$ & $8(4.3)$ & $52(3.1)$ & & $67(3.4)$ \\
\hline
\end{tabular}




\section{Continued}

\begin{tabular}{|c|c|c|c|c|c|}
\hline Fruits n (\%) & & & & & \\
\hline High Consumption & $93(80.9)$ & $161(86.1)$ & $1457(86.0)$ & \multirow{2}{*}{0.309} & $1711(85.7)$ \\
\hline Low Consumption & $22(19.1)$ & $26(13.9)$ & $237(14.0)$ & & $285(14.3)$ \\
\hline \multicolumn{6}{|l|}{ Family History $\mathbf{n}(\%)$} \\
\hline Yes & $31(29.2)$ & $33(19.3)$ & $314(20.0)$ & 0.069 & $378(20.5)$ \\
\hline \multicolumn{6}{|l|}{ Systemic Diagnosis n (\%) } \\
\hline $\mathrm{DM}$ & $11(9.6)$ & $26(13.9)$ & $180(10.6)$ & \multirow{3}{*}{0.329} & $217(10.8)$ \\
\hline SH & $77(66.9)$ & $106(56.7)$ & $1013(59.7)$ & & $1196(59.8)$ \\
\hline $\mathrm{DM} / \mathrm{SH}$ & $27(23.5)$ & $55(29.4)$ & $505(29.7)$ & & $587(29.3)$ \\
\hline \multicolumn{6}{|l|}{ Weight Kg } \\
\hline Median (RIQ) & $70.0(61.7-80.0)$ & $71.0(63.0-80.0)$ & $70.0(62.0-80.0)$ & 0.865 & $70.0(62.0-80.0)$ \\
\hline \multicolumn{6}{|l|}{ Height $\mathrm{cms}$} \\
\hline Median (RIQ) & $162.0(155.0-170.0)$ & $160.0(155.0-167.0)$ & $160.0(154.0-167.0)$ & 0.194 & $160.0(155.0-167.0)$ \\
\hline \multicolumn{6}{|l|}{ BMI } \\
\hline Median (RIQ) & $26.9(23.9-30.2)$ & $26.9(24.6-30.7)$ & $27.2(24.5-30.7)$ & 0.479 & $27.1(24.5-30.7)$ \\
\hline \multicolumn{6}{|c|}{ Abdominal Circumference } \\
\hline Median (RIQ) & $97.0(90.0-104.0)$ & $96.0(88.0-102.0)$ & $96.0(89.0-104.0)$ & 0.628 & $96.0(89.0-104.0)$ \\
\hline \multicolumn{6}{|l|}{ DBP } \\
\hline$<60$ & $18(15.6)$ & $28(15.3)$ & $163(9.7)$ & \multirow{5}{*}{0.003} & $209(10.6)$ \\
\hline $61-70$ & $32(27.8)$ & $50(27.3)$ & $449(26.7)$ & & $531(26.8)$ \\
\hline $71-80$ & $37(32.2)$ & $63(34.4)$ & $714(42.5)$ & & $814(41.1)$ \\
\hline $81-90$ & $15(13.0)$ & $36(19.7)$ & $260(15.5)$ & & $311(15.7)$ \\
\hline$>90$ & $13(11.3)$ & $6(3.3)$ & $94(5.6)$ & & $113(5.7)$ \\
\hline \multicolumn{6}{|l|}{ SBP } \\
\hline$<110$ & $24(20.9)$ & $50(27.3)$ & $321(19.1)$ & \multirow{4}{*}{0.093} & $395(19.9)$ \\
\hline $111-120$ & $31(26.9)$ & $47(25.7)$ & $539(32.1)$ & & $617(31.2)$ \\
\hline $121-140$ & $44(38.3)$ & $66(36.1)$ & $656(39.1)$ & & $766(38.7)$ \\
\hline$>140$ & $16(13.9)$ & $20(10.9)$ & $164(9.7)$ & & $200(10.1)$ \\
\hline \multicolumn{6}{|l|}{ Comorbidities n (\%) } \\
\hline Dyslipidemia & $50(43.4)$ & $83(44.4)$ & $757(44.9)$ & 0.949 & $890(44.8)$ \\
\hline Migraine & $10(8.8)$ & $16(8.6)$ & $186(11.0)$ & 0.530 & $212(10.6)$ \\
\hline Autoimmune dis & $23(21.1)$ & $17(9.7)$ & $191(11.8)$ & 0.015 & $231(12.2)$ \\
\hline Coronary Disease & $17(14.9)$ & 37 (19.9) & $283(16.7)$ & 0.472 & $337(16.9)$ \\
\hline Cáncer & $8(7.0)$ & $6(3.2)$ & $78(4.6)$ & 0.304 & $92(4.6)$ \\
\hline Thyroid disease & $16(14.0)$ & $25(13.4)$ & $392(23.1)$ & 0.001 & $(1.7)$ \\
\hline \multicolumn{6}{|l|}{ IOP } \\
\hline Median (IQR) & $15(12-20)$ & $15(13-17)$ & $14(12-16)$ & 0.001 & $14(12-16)$ \\
\hline \multicolumn{6}{|l|}{$\mathrm{CCT}$} \\
\hline$<500$ & $23(20.2)$ & $28(15.1)$ & $209(12.9)$ & & $260(13.6)$ \\
\hline$>500$ & $91(79.8)$ & $157(84.9)$ & $1406(87.1)$ & 0.076 & $1654(86.4)$ \\
\hline Median (IQR) & $530.0(505.0-551.2)$ & $529.0(509.0-552.5)$ & $534.0(513.0-558.0)$ & & $533.0(512.0-557.0)$ \\
\hline \multicolumn{6}{|l|}{ OPP n (\%) } \\
\hline$<40$ & $28(24.6)$ & $41(22.4)$ & $221(13.2)$ & & $290(14.7)$ \\
\hline $41-50$ & $51(44.7)$ & $82(44.8)$ & $818(48.8)$ & & $951(48.2)$ \\
\hline $51-60$ & $26(22.8)$ & $55(30.0)$ & $541(32.3)$ & 0.000 & $622(31.5)$ \\
\hline$>60$ & $9(7.9)$ & $5(2.7)$ & $96(5.7)$ & & $110(5.6)$ \\
\hline Median (IQR) & $46.5(40.0-51.4)$ & $46.2(40.8-51.9)$ & $47.8(43.2-52.2)$ & & $47.5(42.8-52.2)$ \\
\hline
\end{tabular}

IOP: Intraocular Pressure; CCT: IQR: Interquartile Range; OPP: Ocular perfusion pressure: SBP: Systolic Blood Pressure; DBP: Diastolic Blood Pressure. 
Table 4. Multivariate analysis of the risk factors associated with POAG.

\begin{tabular}{|c|c|c|c|c|}
\hline Variables & $\%$ Confirmed POAG & $\begin{array}{c}\text { Confirmed POAG } \\
\text { OR }[95 \% \text { IC] }\end{array}$ & $\%$ Suspected POAG & $\begin{array}{l}\text { Suspected POAG } \\
\text { OR }[95 \% \text { IC] }\end{array}$ \\
\hline \multicolumn{5}{|l|}{ Age } \\
\hline $50-59$ & $4.09 \%$ & 1 & $10.50 \%$ & 1 \\
\hline $60-69$ & $4.66 \%$ & $1.06[0.57-1.96]$ & $9.33 \%$ & $1.02[0.67-1.55]$ \\
\hline $70-79$ & $8.80 \%$ & $2.55[1.38-4.71]^{\star *}$ & $7.87 \%$ & $0.99[0.60-1.62]$ \\
\hline$>80$ & $6.87 \%$ & $1.88[0.72-4.86]$ & $10.69 \%$ & $1.34[0.63-2.87]$ \\
\hline \multicolumn{5}{|l|}{ Sex } \\
\hline Female & $4.38 \%$ & 1 & $9.23 \%$ & 1 \\
\hline Male & $8.17 \%$ & $1.99[1.22-3.25]^{* *}$ & $9.56 \%$ & $0.95[0.65-1.41]$ \\
\hline \multicolumn{5}{|l|}{ Race } \\
\hline Non White-White(mestizo) & $6.04 \%$ & 1 & $8.46 \%$ & 1 \\
\hline Black & $4.90 \%$ & $0.74[0.27-2.05]$ & $15.69 \%$ & $1.84[0.97-3.48]^{*}$ \\
\hline White & $3.69 \%$ & $0.57[0.25-1.30]$ & $10.33 \%$ & $1.27[0.77-2.08]$ \\
\hline Other & $9.09 \%$ & $0.86[0.24-3.06]$ & $18.18 \%$ & $1.69[0.72-3.94]$ \\
\hline \multicolumn{5}{|l|}{ Marital Status } \\
\hline Other & $5.31 \%$ & 1 & $8.03 \%$ & 1 \\
\hline Married/Free Union & $6.08 \%$ & $1.20[0.73-1.98]$ & $10.24 \%$ & $1.51[1.02-2.23]^{\star}$ \\
\hline \multicolumn{5}{|l|}{ Family History n (\%) } \\
\hline No & $5.11 \%$ & 1 & $9.41 \%$ & 1 \\
\hline Yes & $8.20 \%$ & $1.62[0.98-2.67]^{*}$ & $8.73 \%$ & $0.97[0.63-1.51]$ \\
\hline \multicolumn{5}{|l|}{ Physical Activity n (\%) } \\
\hline Low or inactive & $5.31 \%$ & 1 & $8.31 \%$ & 1 \\
\hline Moderate & $5.85 \%$ & $1.03[0.62-1.71]$ & $10.11 \%$ & $1.44[0.98-2.11]^{*}$ \\
\hline high & $9.49 \%$ & $1.97[0.93-4.15]^{*}$ & $16.06 \%$ & $2.35[1.33-4.16]^{* \star}$ \\
\hline \multicolumn{5}{|l|}{ Processed meat n (\%) } \\
\hline Low Consumption & $5.58 \%$ & 1 & $8.54 \%$ & 1 \\
\hline High Consumption & $6.58 \%$ & $1.22[0.61-2.41]$ & $15.79 \%$ & $1.89[1.18-3.03]^{\star *}$ \\
\hline \multicolumn{5}{|l|}{ DBP mm Hg } \\
\hline$<60$ & $8.61 \%$ & $1.91[0.78-4.67]$ & $13.40 \%$ & $1.12[0.55-2.30]$ \\
\hline $61-70$ & $6.03 \%$ & $1.57[0.85-2.93]$ & $9.42 \%$ & $1.25[0.77-2.05]$ \\
\hline $71-80$ & $4.55 \%$ & 1 & $7.74 \%$ & 1 \\
\hline $81-90$ & $4.82 \%$ & $1.69[0.77-3.70]$ & $11.58 \%$ & $1.96[1.10-3.48]^{\star *}$ \\
\hline$>90$ & $11.50 \%$ & $5.84[1.66-20.52]^{\star *}$ & $5.31 \%$ & $0.60[0.13-2.86]$ \\
\hline \multicolumn{5}{|l|}{ Autoimmune disease } \\
\hline No & $5.16 \%$ & 1 & $9.48 \%$ & 1 \\
\hline Yes & $9.96 \%$ & $2.20[1.24-3.92]^{\star \star}$ & $7.36 \%$ & $0.82[0.45-1.48]$ \\
\hline \multicolumn{5}{|l|}{ Thyroid disease } \\
\hline No & $6.28 \%$ & 1 & $10.32 \%$ & 1 \\
\hline Yes & $3.70 \%$ & $0.47[0.25-0.88]^{* \star}$ & $5.77 \%$ & $0.65[0.40-1.05]^{*}$ \\
\hline \multicolumn{5}{|l|}{ IOP } \\
\hline$<21 \mathrm{~mm} \mathrm{Hg}$ & $4.81 \%$ & 1 & $9.20 \%$ & 1 \\
\hline$\geq 21 \mathrm{~mm} \mathrm{Hg}$ & $34.43 \%$ & $7.18[3.09-16.69]^{* * *}$ & $17.75 \%$ & $1.55[0.59-4.04]$ \\
\hline \multicolumn{5}{|l|}{ CCT } \\
\hline$<500$ & $8.85 \%$ & $1.94[1.09-3.43]^{\star *}$ & $10.77 \%$ & $1.10[0.67-1.81]$ \\
\hline$>500$ & $5.50 \%$ & 1 & $9.49 \%$ & 1 \\
\hline \multicolumn{5}{|l|}{ OPP } \\
\hline$<40$ & $9.66 \%$ & $1.08[0.50-2.31]$ & $14.14 \%$ & $2.23[1.27-3.91]^{\star *}$ \\
\hline $41-50$ & $5.36 \%$ & 1 & $8.62 \%$ & 1 \\
\hline $51-60$ & $4.18 \%$ & $0.62[0.29-1.27]$ & $8.84 \%$ & $0.72[0.41-1.24]$ \\
\hline$>60$ & $8.18 \%$ & $0.41[0.09-1.73]$ & $4.55 \%$ & $0.38[0.08-1.86]$ \\
\hline
\end{tabular}

${ }^{\star} \mathrm{p}<0.10 ;{ }^{* *} \mathrm{p}<0.05 ;{ }^{* * *} \mathrm{p}<0.001$; IOP: Intraocular Pressure; CCT: IQR: Interquartile Range; OPP: Ocular perfusion pressure: SBP: Systolic Blood Pressure; DBP: Diastolic Blood Pressure. 
The proportion of IOP $\geq 21 \mathrm{mmHg}$ was $1.8 \%$ among the patients without glaucoma, $4.8 \%$ in the group with suspected POAG and $18.2 \%$ in the one with confirmed POAG. Figure 3 shows the distribution of IOP according to POAG diagnosis. CCT $<500$ were more frequent in cases with confirmed POAG. Figure 3 shows the distribution of IOP according to diagnosis. The number of cases with low values of OPP $(<40 \mathrm{~mm} \mathrm{Hg})$ was higher in cases with suspected or confirmed POAG compared to the non-glaucoma group (Table 3 and $\mathrm{Ta}$ ble 4).

\subsection{Risk Factors Associated with POAG}

In the multivariate analysis, the risk factors related to glaucoma suspects were high physical activity, high consumption of processed meat, high DBP values and low OPP values. While, older age, male sex, diagnosis of autoimmune disease, IOP $>21 \mathrm{~mm} \mathrm{Hg}$ and CCT $<500$ were associated with the diagnosis of confirmed POAG (Table 4).

\section{Discussion}

This study, to our knowledge, is the first program-based study to report the prevalence and clinical characteristics of glaucoma in subjects over 50 years of age in Colombia. Our study was performed in six cities of the country including patients older than 50 years with $\mathrm{SH}$ and/or DM. The decision to select this age criteria, in addition to adjusting to the central hypothesis of the investigation, was based on efficiency criteria in the design. Specifically, the decision was made by observing that in previous studies the prevalence of glaucoma below 50 years is very low, namely: Barbados, 1.4\% [4], LALES, 1.32\% [10], Project VER, 0.5\% [25], Baltimore $0.18 \%$ [3]; as well as the prevalence of $\mathrm{SH}$ and DM in people younger than 50 years in Colombia [26] [27].

As expected, the prevalence of POAG in our study was 5.6\%, which is higher than those reported in most studies [1]-[8] [10] [25]. This finding is due to the fact that our population corresponded to diabetic and/or hypertensive patients, unlike other previous population-based studies where the only inclusion criteria was age (over 40 years), independently of any systemic disease. It is noteworthy that the prevalence of glaucoma in our Latino DM/SH population almost equals the observed 7\% prevalence among the African-descendant population in the Barbados study [4]. Also, is important to notice that the prevalence of POAG could be similar between Latinos and African-descendant patients. Future studies in a wider and more representative latino population will help us better discern the similarities and differences with African-descendant populations.

The prevalence of POAG in our study among individuals 70 - 79 years old was 8.6\% [6.5 - 11.3], while in those with 50 to 59 years old was 4.0\% [2.7 - 5.9]. Our study, reflect an age-related increase in prevalence of POAG, similar observations have been reported previously in other prevalence studies [10] [25]. 


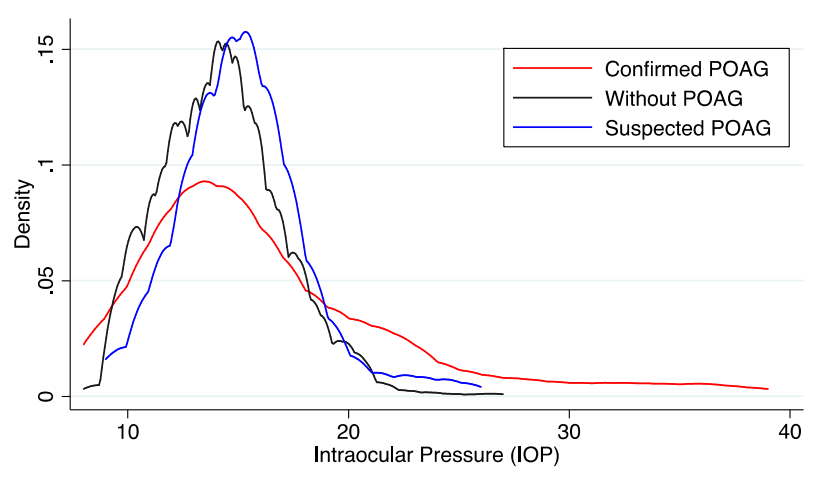

Figure 3. Intraocular pressure distribution curve according to diagnosis.

Our results are worrisome regarding the poor knowledge of the diagnosis in this group of patients with $\mathrm{SH}$ and DM (more than $75 \%$ were unaware), since the late diagnosis of glaucoma can lead to severe visual impairment. A similar situation was described in the LALES study, over $75 \%(n=220)$ of the 291 persons with POAG had no history of glaucoma or treatment for glaucoma. Similarly, over $75 \%(n=165)$ of those diagnosed with ocular hypertension were previously undiagnosed [10]. Other similar rates where described in Northern Italy (78\%) [20] and are higher than those found in other population-based studies in Melbourne (50\%) [7], Blue Mountains (51\%) [6], Baltimore (58\% in blacks and $50 \%$ in non-Hispanic whites) [3], Rotterdam (53\%) [5], Barbados (51\%) [4], and Arizona (62\%) [25].

Costa et al. performed a comparative study among 183 patients interviewed at the Wills Eye Hospital (Philadelphia, USA) and 100 patients from the Glaucoma Service at the University of Campinas (Campinas, Brazil). Linear regression analysis showed a positive relationship between level of education and knowledge about glaucoma (r: 0.65, p: 0.001). This study concludes that knowledge about glaucoma significantly varies in an urban population when one is located in a high-income country (US), where one third had no knowledge of the disease, compared to a middle-income country (Brazil) in which two-thirds had no knowledge of the disease [28].

The lack of awareness of the disease should influence decision making across the health system to implement effective and permanent strategies for the early detection of the disease with the aim of facilitating that patients with risk factors have access to a timely treatment. This situation is more alarming if we take into account that patients from $\mathrm{SH}$ and DM programs participating in this study are supposed to be routinely evaluated by doctors and nurses to control underlying disease. This is the best scenario, since $30 \%-50 \%$ of patients with DM are unaware of their disease or do not have access to such programs [27].

A trend for a higher prevalence of POAG in those with more than 5 years of being diagnosed with DM and/or SH was observed (Figure 2). Similar to our results, The Los Angeles Latino Study (LALES) LALES reported 5894 participants, 1157 (19.6\%) had DM type 2 and 288 (4.9\%) had POAG. The prevalence 
of POAG $40 \%$ higher in participants with DM than in those without DM (age/gender/intraocular pressure-adjusted odds ratio, 1.4; 95\% confidence interval, 1.03 - 1.8; $\mathrm{p}$ value 0.03 ). Trend analysis revealed that a longer duration of $\mathrm{DM}$ (stratified into 5-year increments) was associated with a higher prevalence of POAG $(\mathrm{p}<0.0001)$ [12].

To the best of our knowledge, this is the first population based study to report this independent association between longer duration of DM and higher prevalence of POAG in a latino population outside the US. Additionally, a systematic review by Minwen et al. of DM as a risk factor for POAG, including thirteen studies-seven case-control studies and six population-based cohort studies-reported a pooled RR of the association between DM and POAG, based on the risk estimates of the six cohort studies, to be 1.40 (95\% CI, 1.25 - 1.57) [29].

DM may affect vascular autoregulation of the retina and optic nerve causing microvascular damage. Vascular disturbances to the anterior portion of the optic nerve are postulated to be at least partially responsible for optic nerve head changes, which can result in glaucomatous optic neuropathy [30] [31] [32]. Additionally, $\mathrm{DM}$ also compromises glial and neuronal functions and metabolism in the retina, which can make retinal neurons, including retinal ganglion cells, more susceptible to glaucomatous damage [33]. The higher prevalence of POAG in subjects with longer duration of DM might be related to a prolonged insult to the retina and optic nerve via vascular, glial, and neuronal factors.

Similar to DM, SH diagnosed for more than 5 years was also related to higher prevalence of POAG. Additionally, elevated DBP showed a tendency to be positively related to POAG. Specifically, the prevalence of POAG was approximately four-fold among the subjects with DBP $>90 \mathrm{~mm} \mathrm{Hg}$ as compared to those with a DBP between 71 and $80 \mathrm{~mm} \mathrm{Hg}$. In SH, an endothelial dysfunction is observed initially due to the alteration in endothelin, which leads to a change in the size of the arterioles, then an increase in resistance to blood flow, reduction of perfusion and finally a loss of vascular autoregulation [34].

The Los Angeles Latino Study (LALES) evidenced that patients with SBP greater than $160 \mathrm{~mm} \mathrm{Hg}$ and those with MAP greater than $110 \mathrm{~mm} \mathrm{Hg}$ had a higher prevalence of POAG (OR 2.0 and 1.6, respectively). In addition, patients with a DBP of less than $60 \mathrm{~mm} \mathrm{Hg}$ had a higher prevalence of POAG (OR: 1.9) [21]. This bimodal relationship between arterial pressure and glaucoma evidenced in LALES was also seen in our study.

Our study included two important vascular risk factors for glaucoma. The implementation of standardized protocols for conducting the study makes the information collected from the six participating cities comparable, increasing the quality of the information. Furthermore, given that the sociodemographic and risk factors surveys were performed before the ophthalmologic evaluation, this would reduce the presence of a differential information bias between patients diagnosed with suspicious or confirmed POAG in comparison with healthy subjects.

The suspected and confirmed case definition of POAG was based on international criteria used by other population studies [24], which facilitates the com- 
parison of results. Also, the diagnosis of glaucoma was based on optic disc and VF criteria, independent of IOP level with a standardized criterion.

On the other hand, a potential weakness of our research is that individuals were directly enrolled at $\mathrm{SH}$ and DM programs. This could imply these patients represent a modified cohort, due to changes induced in their habits and lifestyles according to recommendations made at programs designed for them. So asymptomatic and non-diagnosed SH/DM individuals could have an even higher prevalence of glaucoma. Due to the cross-sectional design of our study, it is not possible to conclusively establish causal associations. However, these findings represent a starting point for further studies that attempt to evaluate the temporal association between the factors evaluated and glaucoma in patients with SH and/or DM. Another potential weakness is the fact that it is impossible to know if patients who have already undergone cataract surgery had developed neuropathy before or after surgery, and that there may probably be cases that originally had chronic closed angles or at least narrow or intermittent closures.

\section{Conclusions}

In summary, the high prevalence of POAG in patients with SH and DM in a latino population and the high unawareness of the disease present public health implications in Colombia and Latin America. Future studies have to be carried out to generalize these findings to the entire population with and without risk factors.

This study should be a basis to create a public health policy where all DM and SH patients have a referral to a complete ophthalmological evaluation at least once a year.

\section{Ethical Statements}

The Universidad del Valle Review Board approved this study (Approval Code 030-014), and all participants signed informed consent. This research was conducted according to the Declaration of Helsinki.

\section{Acknowledgements}

The authors would like to thank Tecnoquimicas S.A. for their financial support for this study. The funder had no role in the design, data collection, analysis and interpretation of the data, nor on the writing of the study.

\section{Conflicts of Interest}

The authors declare no conflicts of interest regarding the publication of this paper.

\section{References}

[1] Quigley, H. and Broman, T. (2006) The Number of People with Glaucoma Worldwide in 2010 and 2020. British Journal of Ophthalmology, 90, 262-267.

https://doi.org/10.1136/bjo.2005.081224 
[2] Klein, R., Klein, B.E., Linton, K.L. and De Mets, D.L. (1991) The Beaver Dam Eye Study: Visual Acuity. Ophthalmology, 98, 1310-1315. https://doi.org/10.1016/S0161-6420(91)32137-7

[3] Tielsch, J.M., Sommer, A., Witt, K., Katz, J. and Royall, R.M. (1990) Blindness and Visual Impairment in an American Urban Population. The Baltimore Eye Survey. Archives of Ophthalmology (Chicago, Ill 1960), 108, 286-290. https://doi.org/10.1001/archopht.1990.01070040138048

[4] Hyman, L., Wu, S.Y., Connell, A.M., et al. (2001) Prevalence and Causes of Visual Impairment in The Barbados Eye Study. Ophthalmology, 108, 1751-1756. https://doi.org/10.1016/S0161-6420(01)00590-5

[5] Klaver, C.C., Wolfs, R.C., Vingerling, J.R., Hofman, A. and de Jong, P.T. (1998) Age-Specific Prevalence and Causes of Blindness and Visual Impairment in an Older Population: The Rotterdam Study. Archives of Ophthalmology (Chicago, Ill 1960), 116, 653-658. https://doi.org/10.1001/archopht.116.5.653

[6] Mitchell, P., Smith, W., Chey, T. and Healey, P.R. (1997) Open-Angle Glaucoma and Diabetes. The Blue Mountains Eye Study, Australia. Ophthalmology, 104, 712-718. https://doi.org/10.1016/S0161-6420(97)30247-4

[7] Wensor, M.D., McCarty, C.A., Stanislavsky, Y.L., Livingston, P.M. and Taylor, H.R. (1998) The Prevalence of Glaucoma in the Melbourne Visual Impairment Project. Ophthalmology, 105, 733-739. https://doi.org/10.1016/S0161-6420(98)94031-3

[8] Munoz, B., West, S.K., Rubin, G.S., et al. (2000) Causes of Blindness and Visual Impairment in a Population of Older Americans: The Salisbury Eye Evaluation Study. Archives of Ophthalmology (Chicago, Ill 1960), 118, 819-825.

https://doi.org/10.1001/archopht.118.6.819

[9] Kreft, D., Doblhammer, G., Guthoff, R.F. and Frech, S. (2019) Prevalence, Incidence, and Risk Factors of Primary Open-Angle Glaucoma: A Cohort Study Based on Longitudinal Data from a German Public Health Insurance. BMC Public Health, 19, 851. https://doi.org/10.1186/s12889-019-6935-6

[10] Varma, R., Francis, B.A., et al. (2004) Prevalence of Open-Angle Glaucoma and Ocular Hypertension in Latinos: The Los Angeles Latino Eye Study. Ophthalmology, 111, 1439-1448. https://doi.org/10.1016/j.ophtha.2004.01.025

[11] Kim, K.E., Kim, M.J., Park, K.H., et al. (2016) Prevalence, Awareness, and Risk Factors of Primary Open-Angle Glaucoma: Korea National Health and Nutrition Examination Survey 2008-2011. Ophthalmology, 123, 532-541. https://doi.org/10.1016/j.ophtha.2015.11.004

[12] Chopra, V., Varma, R., Francis, B.A., Wu, J., Torres, M. and Azen, S.P. (2008) Type 2 Diabetes Mellitus and the Risk of Open-Angle Glaucoma. The Los Angeles Latino Eye Study. Ophthalmology, 115, 227-233. https://doi.org/10.1016/j.ophtha.2007.04.049

[13] Klein, B.E., Klein, R. and Jensen, S.C. (1994) Open-Angle Glaucoma and Older-Onset Diabetes. The Beaver Dam Eye Study. Ophthalmology, 101, 1173-1177. https://doi.org/10.1016/S0161-6420(94)31191-2

[14] Dielemans, I., de Jong, P.T., Stolk, R., Vingerling, J.R., Grobbee, D.E. and Hofman, A. (1996) Primary Open-Angle Glaucoma, Intraocular Pressure, and Diabetes Mellitus in the General Elderly Population. The Rotterdam Study. Ophthalmology, 103, 1271-1275. https://doi.org/10.1016/S0161-6420(96)30511-3

[15] Song, B.J., Aiello, L.P. and Pasquale, L.R. (2016) Presence and Risk Factors for Glaucoma in Patients with Diabetes. Current Diabetes Reports, 16, 124.

https://doi.org/10.1007/s11892-016-0815-6 
[16] Zhao, Y.X. and Chen, X.W. (2017) Diabetes and Risk of Glaucoma: Systematic Review and a Meta-Analysis of Prospective Cohort Studies. International Journal of Ophthalmology, 10, 1430-1435.

[17] Kahn, H.A. and Milton, R.C. (1980) Alternative Definitions of Open-Angle Glaucoma. Effect on Prevalence and Associations in the Framingham Eye Study. Archives of Ophthalmology (Chicago, Ill 1960), 98, 2172-2177. https://doi.org/10.1001/archopht.1980.01020041024003

[18] Bankes, J.L. (1967) Ocular Tension and Diabetes Mellitus. British Journal of Ophthalmology, 51, 557-561. https://www.ncbi.nlm.nih.gov/pubmed/6035958 https://doi.org/10.1136/bjo.51.8.557

[19] Tielsch, J.M., Katz, J., Quigley, H.A., Javitt, J.C. and Sommer, A. (1995) Diabetes, Intraocular Pressure, and Primary Open-Angle Glaucoma in the Baltimore Eye Survey. Ophthalmology, 102, 48-53. https://doi.org/10.1016/S0161-6420(95)31055-X

[20] Bonomi, L., Marchini, G., Marraffa, M., Bernardi, P., Morbio, R. and Varotto, A. (2000) Vascular Risk Factors for Primary Open Angle Glaucoma: The Egna-Neumarkt Study. Ophthalmology, 107, 1287-1293. https://doi.org/10.1016/S0161-6420(00)00138-X

[21] Memarzadeh, F., Chung, J., Azen, S.P., Varma, R., et al. (2010) Blood Pressure, Perfusion Pressure, and Open-Angle Glaucoma: The Los Angeles Latino Eye Study. Investigative Ophthalmology \& Visual Science, 51, 2872-2877. https://doi.org/10.1167/iovs.08-2956

[22] American Diabetes Association (2010) Diagnosis and Classification of Diabetes Mellitus. Diabetes Care, 33, S62-S69. https://doi.org/10.2337/dc10-S062

[23] Mancia, G., Fagard, R., Narkiewicz, K., et al. (2013) ESH/ESC Guidelines for the Management of Arterial Hypertension: The Task Force for the Management of Arterial Hypertension of the European Society of Hypertension (ESH) and of the European Society of Cardiology (ESC). European Heart Journal, 34, 2159-2219. https://doi.org/10.1093/eurheartj/eht151

[24] Foster, P.J., Buhrmann, R., Quigley, H. and Johnson, G.J. (2002) The Definition and Classification of Glaucoma in Prevalence Surveys. British Journal of Ophthalmology, 86, 238-243. https://doi.org/10.1136/bjo.86.2.238

[25] Quigley, H., West, S.K., Rodriguez, J., Munoz, B., Klein, R. and Snyder, R. (2001) The Prevalence of Glaucoma in a Population-Based Study of Hispanic Subjects: Proyecto VER. Archives of Ophthalmology, 119, 1819-1826. https://doi.org/10.1001/archopht.119.12.1819

[26] Bautista, L.E., Vera-Cala, L.M., Villamil, L., Silva, S.M., Peña, I.M. and Luna, L.V. (2002) Factores de riesgo asociados con la prevalencia de hipertensión arterial en adultos de Bucaramanga, Colombia. Salud Pública de México, 44, 399-405. https://doi.org/10.1590/S0036-36342002000500003

[27] Vargas-Uricoechea, H. and Casas-Figueroa, L.A. (2015) An Epidemiologic Analysis of Diabetes in Colombia. Annals of Global Health, 81, 742-753. https://doi.org/10.1016/j.aogh.2015.11.001

[28] Costa, V.P., Spaeth, G.L., Smith, M., Uddoh, C., Cabral Vasconcellos, J.P. and Kara-José, N. (2006) Patient Education in Glaucoma: What Do Patients Know about Glaucoma? Arquivos Brasileiros de Oftalmologia, 69, 923-927. https://doi.org/10.1590/S0004-27492006000600024

[29] Zhou, M., Wang, W., Huang, W. and Zhang, X. (2014) Diabetes Mellitus as a Risk Factor for Open-Angle Glaucoma: A Systematic Review and Meta-Analysis. PLoS ONE, 9, e102972. https://doi.org/10.1371/journal.pone.0102972 
[30] Yanagi, M., Kawasaki, R., Wang, J.J., Wong, T.Y., Crowston, J. and Kiuchi, Y. (2011) Vascular Risk Factors in Glaucoma: A Review. Clinical and Experimental Ophthalmology, 39, 252-258. https://doi.org/10.1111/j.1442-9071.2010.02455.x

[31] Gerber, A.L., Harris, A., Siesky, B., et al. (2015) Vascular Dysfunction in Diabetes and Glaucoma. Journal of Glaucoma, 24, 474-479. https://doi.org/10.1097/IJG.0000000000000137

[32] Sayin, N., Kara, N. and Pekel, G. (2015) Ocular Complications of Diabetes Mellitus. World Journal of Diabetes, 15, 562-566. https://doi.org/10.4239/wjd.v6.i1.92

[33] Kanamori, A., Nakamura, M., Mukuno, H., Maeda, H. and Negi, A. (2004) Diabetes Has an Additive Effect on Neural Apoptosis in Rat Retina with Chronically Elevated Intraocular Pressure. Current Eye Research, 28, 47-54. https://doi.org/10.1076/ceyr.28.1.47.23487

[34] Anderson, D.R. (1999) Introductory Comments on Blood Flow Autoregulation in the Optic Nerve Head and Vascular Risk Factors in Glaucoma. Survey of Ophthalmology, 43, S5-S9. https://doi.org/10.1016/S0039-6257(99)00046-6 